\title{
The CLASI, a validated tool for the evaluation of skin disease in lupus erythematosus: a narrative review
}

\author{
Srita Chakka ${ }^{1,2 \#} \wedge$, Rebecca L. Krain ${ }^{1,2 \#}$, Josef Symon S. Concha ${ }^{1,2}$, Benjamin F. Chong ${ }^{3}$, \\ Joseph F. Merola ${ }^{4}$, Victoria P. Werth ${ }^{1,2}$ \\ ${ }^{1}$ Corporal Michael J. Crescenz VAMC, Philadelphia, PA, USA; ${ }^{2}$ Department of Dermatology, University of Pennsylvania, Philadelphia, PA, USA; \\ ${ }^{3}$ Department of Dermatology, University of Texas Southwestern Medical Center, Dallas, TX, USA; ${ }^{4}$ Department of Dermatology and Department of \\ Medicine, Division of Rheumatology, Harvard Medical School, Brigham and Women's Hospital, Boston, MA, USA \\ Contributions: (I) Conception and design: VP Werth, BF Chong, JF Merola; (II) Administrative support: VP Werth; (III) Provision of study materials \\ or patients: VP Werth, JSS Concha, S Chakka, RL Krain; (IV) Collection and assembly of data: S Chakka, RL Krain; (V) Data analysis and \\ interpretation: All authors; (VI) Manuscript writing: All authors; (VII) Final approval of manuscript: All authors. \\ "These authors contributed equally to this work. \\ Correspondence to: Victoria P. Werth, MD. Department of Dermatology, Perelman Center for Advanced Medicine, Suite 1-330A, 3400 Civic Center \\ Boulevard, Philadelphia, PA 19104, USA. Email: werth@pennmedicine.upenn.edu.
}

\begin{abstract}
Cutaneous lupus erythematosus (CLE) can present with or without features of systemic lupus erythematosus (SLE), with estimates of the incidence of isolated skin disease almost equaling the incidence of those with systemic disease. However, despite the impact CLE has on a patient's quality of life (QoL), there has been no US Food and Drug Administration (FDA) approved treatment for the disease in the past 50 years. In addition, patients with skin predominant LE are often excluded from clinical SLE trials. In the rare trials that include patients with skin predominant LE, disease activity and progression in the skin are often difficult to evaluate using multi-organ outcome measures. The need for new therapies for CLE and the lack of focus on skin outcomes has led to the development of the Cutaneous Lupus Disease Area and Severity Index (CLASI), a validated organ-specific outcome measure that is not only responsive to change in disease activity and damage but also correlated to changes in a patient's QoL. This paper will emphasize the extensive validation studies performed in developing the CLASI, as well as the importance of clinical trials using the CLASI to address the need for improved therapies for patients with lupus skin manifestations.
\end{abstract}

Keywords: Cutaneous lupus erythematosus (CLE); outcome measures; autoimmune skin disease; validation study

Submitted Jul 10, 2020. Accepted for publication Sep 21, 2020.

doi: $10.21037 /$ atm-20-5048

View this article at: http://dx.doi.org/10.21037/atm-20-5048

\section{Introduction}

Cutaneous disease is the most frequent manifestation of systemic lupus erythematosus (SLE) (1). It is often the first presenting sign and one of the most common complaints of patients suffering from SLE, occurring in approximately $70 \%$ of patients $(1,2)$. Cutaneous lupus erythematosus (CLE) is a potentially scarring, disfiguring process with profound impact on a patient's quality of life (QoL) $(1,3)$. Approximately $10 \%$ of patients with CLE are refractory to all therapies, and approximately $50 \%$ of patients needing systemic therapy require escalation beyond topical and antimalarial therapy to immunosuppressive or biologic therapies to control their skin disease $(4,5)$. CLE can be categorized into acute CLE (ACLE), subacute CLE (SCLE), and chronic CLE (CCLE) (3). CCLE includes

^ ORCID: Srita Chakka, 0000-0001-7288-2346; Rebecca L. Krain, 0000-0002-0002-8973; Josef Symon S. Concha, 0000-0002-4421-1147. 
discoid lupus erythematosus (DLE), lupus erythematosus profundus (LEP), chilblain cutaneous lupus (CHLE), and lupus tumidus (LET) (6).

First-line treatment for CLE includes photoprotection and antimalarial agents (7). Hydroxychloroquine is the preferred antimalarial agent due to its efficacy and tolerability; however, this agent, as well as chloroquine, is associated with retinopathy and patients on either medication should receive regular eye examinations (7). Quinacrine, another antimalarial agent, can be used with hydroxychloroquine, and has rare skin reactions, such as yellowing of the skin $(4,6,7)$. Although antimalarials are generally well-tolerated and provide significant relief to a large subset of patients, quinacrine has unfortunately become largely unavailable due to import restrictions on the powder that was subsequently compounded. For patients unable to tolerate antimalarials, or in recalcitrant cases, other systemic agents (i.e., mycophenolate mofetil, azathioprine, methotrexate, dapsone, sulfasalazine, oral retinoids, thalidomide/lenalidomide, and systemic corticosteroids) should be considered (8). Although these medications can be effective, they can have significant side effects, making it difficult for providers to prescribe these medications $(2,8-10)$.

A literature search of the PubMed database was performed through November 2018. Cutaneous Lupus Disease Area and Severity Index (CLASI) external validation studies were included as well as significant retrospective studies of prospectively collected data that evaluated its correlation to changes in disease activity and QoL. We also included randomized controlled trials that evaluated novel SLE therapies, specifically those that included CLE patients and utilized the CLASI. Prior systematic reviews were considered additional references.

The objective of this narrative review is to highlight the importance of the CLASI, a validated and responsive clinical tool that quantifies cutaneous activity and damage in CLE; address the necessity for improved therapies for CLE; and emphasize the need for a regulatory pathway that will allow for the development of therapies directed at the skin.

We present the following article in accordance with the Narrative Review reporting checklist (available at http:// dx.doi.org/10.21037/atm-20-5048).

\section{A disease severity index for CLE}

In 2003, the Food and Drug Administration (FDA) released a guidance document for the development of new drugs for SLE, suggesting that organ-specific therapies may be submitted to the FDA for approval. These guidelines emphasized the importance of documenting the impact of treatment on disease activity and damage (11). The skin is a visible and important component of SLE, allowing for ongoing observation of activity and damage. More importantly, and sometimes easily forgotten, is the impact that skin disease has on patients.

Several validated instruments have been available to measure and evaluate disease activity for SLE since the 1980 s, and many of them are used in clinical trials to monitor and classify clinical responses to treatment (12-14). However, a number of scores like the SLE Disease Activity Index (SLEDAI) (15), the Lupus Activity Criteria Count (LACC) (16), and the Systemic Lupus Collaborating Clinics/American College of Rheumatology Damage Index for SLE (SLICC/ACR Damage Index for SLE) (17), only document the presence or absence of signs, such as skin rash. Among the 60 outcome measures available for SLE, none are adequate enough to evaluate disease activity in CLE (18).

There are numerous outcome instruments for other inflammatory skin diseases, including psoriasis and atopic eczema $(19,20)$. However, none of them have been suitable for CLE, including the Dermatology Index of Disease Severity (DIDS), which was designed as an universal outcome instrument for skin diseases $(21,22)$. The characteristics that set CLE apart from other dermatologic conditions preclude the use of the same outcome instruments used to evaluate other diseases. These differences are important to recognize and evaluate, since CLE is a heterogeneous disease with various subtypes. The lack of an appropriate and validated outcome measure to evaluate the response in therapeutic trials for CLE prompted the development of the CLASI, with input from the international community of dermatologists and patients (23).

About $15-20 \%$ of patients with CLE have more than one subtype, some of which are more likely than others to cause scarring or dyspigmentation (Figures 1 and 2) (24,25). In one study classifying cutaneous manifestations in 191 patients, it was found that $29 \%$ of patients had two types of CLE and $3 \%$ had three types (26). The CLASI was designed to capture most subtypes except for the rare manifestations of CLE, such as lupus panniculitis or bullous lupus. Since CLE is a disease that can present with multiple morphologies or change subtypes, it is important that a disease instrument be responsive and monitor disease progression over time.

The CLASI, like many outcome measures for SLE and 

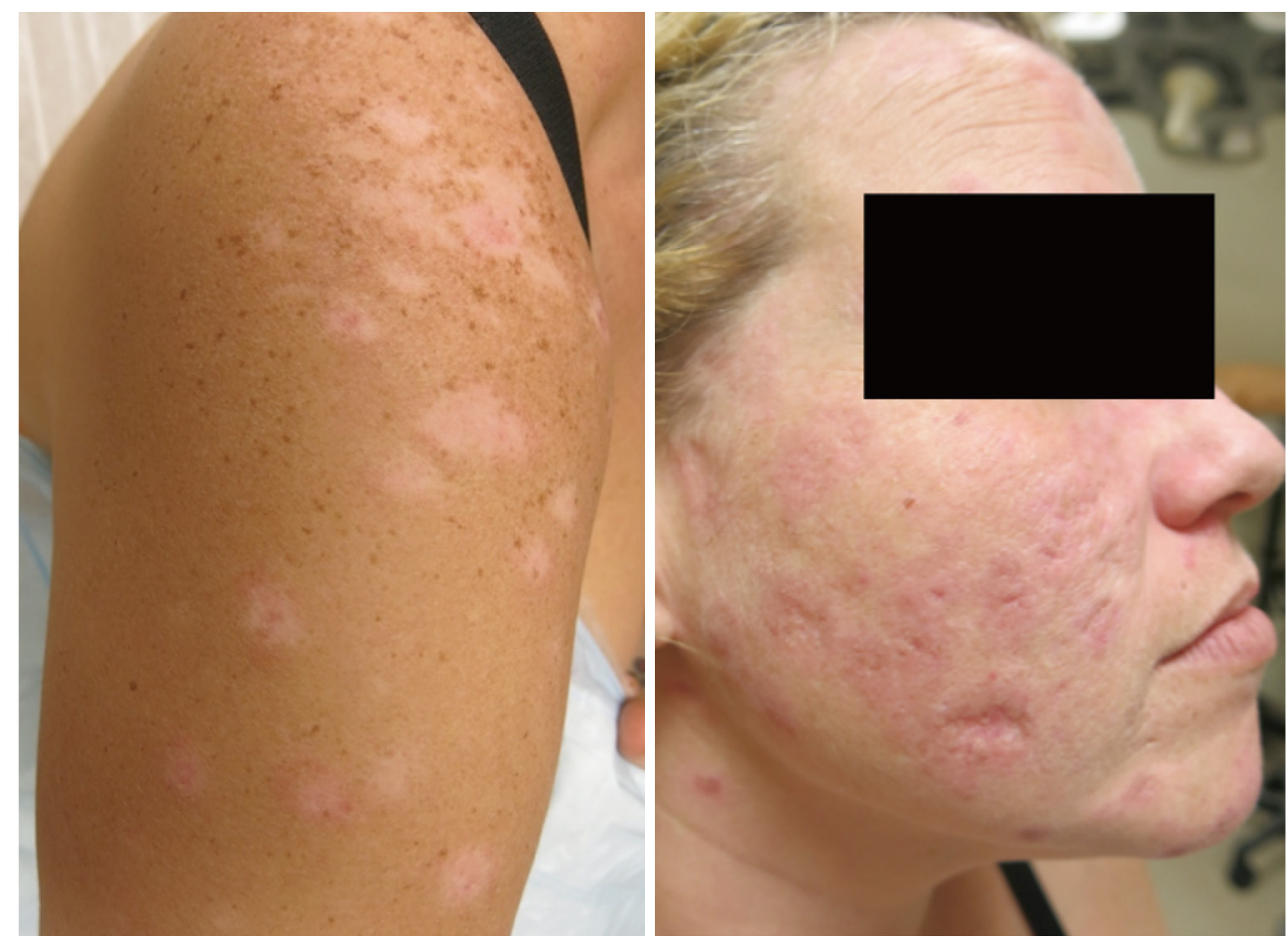

Figure 1 CLE/SLE presenting with both DLE and SCLE. CLE, cutaneous lupus erythematosus; SLE, systemic lupus erythematosus; DLE, discoid lupus erythematosus; SCLE, subacute cutaneous lupus erythematosus.
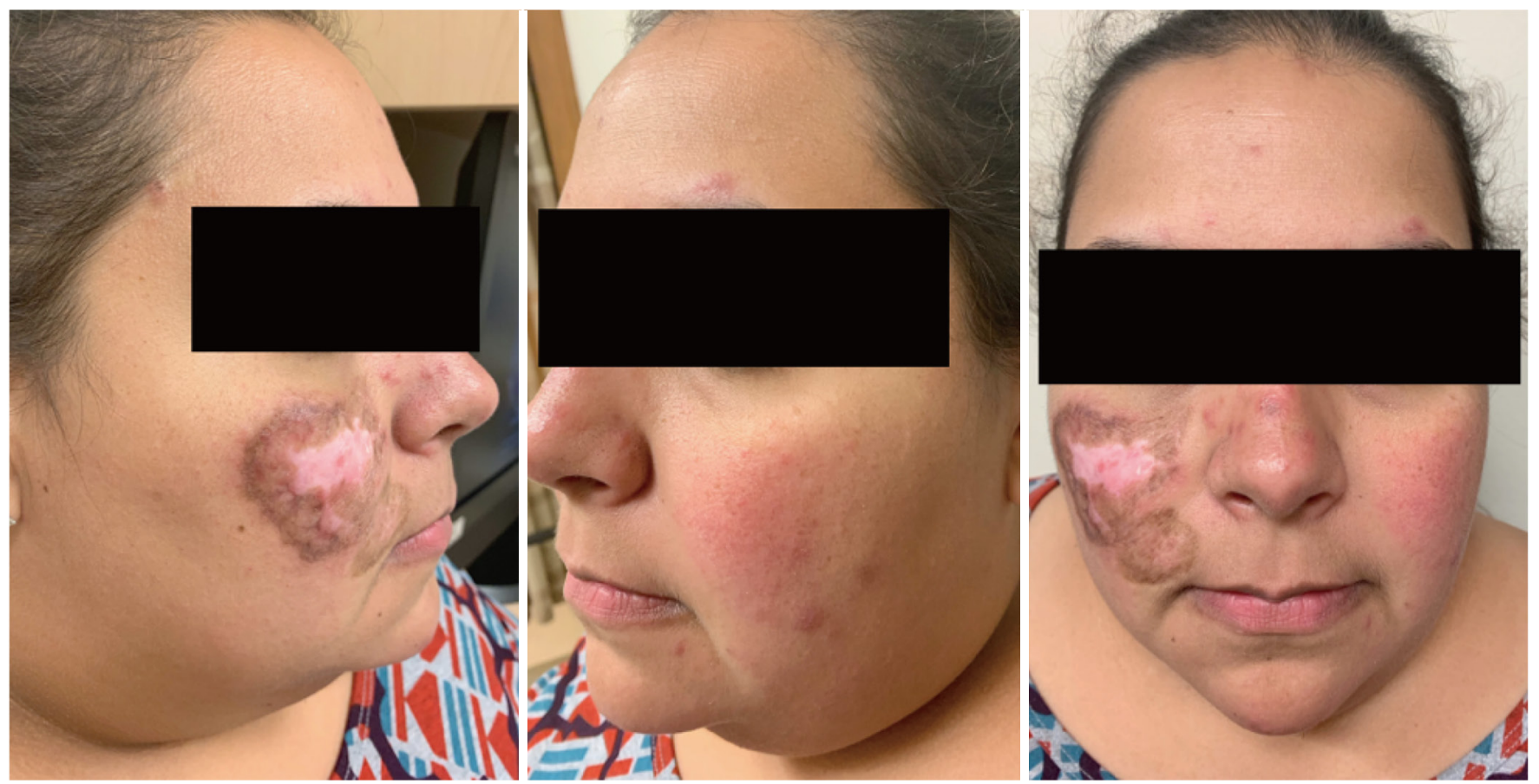

Figure 2 CLE/SLE presenting with both ACLE and DLE. CLE, cutaneous lupus erythematosus; SLE, systemic lupus erythematosus; ACLE, acute cutaneous lupus erythematosus; DLE, discoid lupus erythematosus. 
as mandated by the FDA, has two scores: disease activity and disease-induced damage. Disease activity is scored to a maximum of 70 points and measures erythema, scale/ hypertrophy, mucous membrane involvement, hair loss in the past 30 days, and non-scarring alopecia. Damage is scored to a maximum of 80 points and takes into consideration the presence of dyspigmentation and scarring, including scarring alopecia. The dyspigmentation score is doubled when most of the dyspigmentation has been present for more than 12 months.

Scores are assigned based on anatomic area and the most severe attributes within that area. Affected areas are weighted equally regardless of the surface area and the number of lesions present. Surface area is frequently small, difficult to assess reliably, and does not reflect the true impact of the disease. Lesion counting may paradoxically result in higher numbers as lesions heal and break into smaller lesions. The CLASI separates highly photo exposed and cosmetically sensitive areas such as the face, $V$-neck area, scalp, and extensor surfaces of the arms into a number of distinct categories, effectively weighing exposed areas more prone to develop CLE lesions more heavily in the total scores (27). For example, the head is subdivided into the scalp, ears, nose/malar area, and the rest of face. Each anatomic subdivision individually carries identical weight as anatomically larger areas of the body, such as the back/buttocks and abdomen. This allows the CLASI to account for the effect of anatomic areas that are more affected in CLE, which has been validated with the correlation of QoL with the CLASI activity score $(3,28,29)$. Using severity and responsiveness analyses, it was determined that a higher numerical score indicates more severe disease, with mild, moderate, and severe disease corresponding to a CLASI activity score of 0 to 9,10 to 20 , and 21 to 70, respectively (30). Therefore, a reduction in disease activity is reflected by a reduction in CLASI, which indicates clinical improvement in one or more of these clinical categories: erythema, scale/hypertrophy, mucous membrane involvement, and alopecia.

Erythema is a hallmark in many cutaneous diseases and is used by the CLASI to partly reflect the disease activity of CLE. The scale used in the CLASI is similarly structured in other disease indices, such as the Psoriasis Area and Severity Index (PASI), a gold standard used for evaluation of psoriasis in clinical trials $(19,31,32)$.

Erythema has been proven to be a reliable sign of disease activity by directly reflecting the hyperemia that accompanies inflammation (23). In severe cases, the inflammation may even result in hemorrhagic crusting (33). Erythema can also be transient, as with acute LE where skin lesions may resolve due to background steroid therapy. It has been found that trained visual clinical assessments of erythema were correlated with objective measurements of erythema using a laser Doppler flow meter and a chromameter $(23,34)$.

Erythema can sometimes be difficult to distinguish from background telangiectatic change in a setting of atrophy and may be dependent on a clinician's expertise, and dermoscopy can be helpful in some cases. Despite the relative ease of visual assessment, concerns regarding the difficulty in appreciating varying degrees of erythema in darker skin tones have been noted. However, a study of patients with atopic dermatitis found that skin color did not alter the perception of erythema amongst trained raters (35).

Patients are more frequently concerned with the erythema and scale of disease activity than with the damage resulting from the scarring and dyspigmentation, a finding further confirmed in discussions with many patients (33). The CLASI includes and evaluates signs that are important to patients, as validated by the correlation of QoL with the CLASI activity score $(3,28,29,33)$.

\section{Validation and responsiveness of the CLASI}

The CLASI has demonstrated excellent content and construct validity, inter-rater validity, intra-rater validity and practical applicability. Content validity of the CLASI was assessed and confirmed via a series of standardized interviews with a group of seven dermato-rheumatologists with expertise in CLE and the "American College of Rheumatology Response Criteria Committee on SLE," as well as interviews with patients about attributes of the disease most concerning to them $(23,27)$. Construct validity was confirmed by a strong correlation between the CLASI activity score and the mucocutaneous score of the SLEDAI and between the CLASI damage score and the SLE Damage Index (SDI) skin domain score (36).

The CLASI was validated with dermatologists and dermatology residents, and validation was extended to rheumatologists $(27,37)$. The CLASI demonstrated suitability for use in multicenter trials as there was excellent inter- and intra-rater reliability of both the activity and damage scores of the CLASI, as measured by the intra-class correlation coefficient (27). In addition, the CLASI was recently evaluated in pediatric lupus and was determined to be a reliable and valid outcome measure for the assessment 
of pediatric CLE (38). One study showed the CLASI had both convergent and discriminant validity when compared to the SLEDAI and SDI, and responsiveness. Children with pediatric CLE who had a reduction of CLASI activity by $50 \%$ between study visits had significant improvement in QoL (39).

To determine whether the CLASI was reflective of changes in disease activity, the CLASI scores were correlated with changes in clinical outcome measures using 0 to 10 visual analog scales, such as the patient's visual analog scale $\left(\mathrm{r}_{\mathrm{p}}=0.85, \mathrm{P}=0.007, \mathrm{n}=8\right)$, and the patient's assessment of pain and itch $\left(\mathrm{r}_{\mathrm{p}}=0.98, \mathrm{P}=0.004, \mathrm{n}=5\right)(40,41)$. Other studies have validated the clinical responsiveness of the CLASI in patients with SCLE; activity scores in patients with tumid LE were found to decrease significantly after three months of therapy with an antimalarial medication (42). CLASI activity scores were also found to decrease significantly after three months of therapy with mycophenolate sodium, which correlated with improvements on ultrasound and colorimetry (43). It has been demonstrated that CLASI activity scores in patients with DLE decrease significantly after 6-18 weeks of pulsed dye laser therapy (44). Studies have also confirmed a similar response of the CLASI after various therapeutic interventions with hydroxychloroquine, thalidomide, apremilast, lenalidomide, and IVIG (45-51).

As with any instrument used to measure disease activity and damage, a relative degree of training and knowledge of disease entity is required to ensure proper evaluation of a patient. The CLASI was developed with the involvement of expert dermatologists and rheumatologists and with interviews of patients about their concerns related to CLE. In addition, the scores were also correlated to patients' assessments of disease activity and damage. The CLASI was developed prior to the January 2014 FDA guidance on a Qualification Process for Drug Development Tools when rheumatology at the FDA voiced a need for organ-specific outcome measures. Most tools used in clinical trials have not been evaluated through this process or undergone the rigorous development process performed with the CLASI.

In May 2018, the $4^{\text {th }}$ International Conference on Cutaneous Lupus Erythematosus (ICCLE) was held in conjunction with the International Investigative Dermatology Meeting. There was $100 \%$ agreement amongst leading dermatologists and rheumatologists that the CLASI is the preferred outcome measure to evaluate skin disease severity in CLE (52).

\section{CLASI and biomarkers in CLE}

Studies have shown a correlation between the CLASI and biomarkers of inflammation $(53,54)$. Type I interferons (IFN), for instance, have a role in the development of CLE, and type I IFN-regulated proteins have been found to have an increased expression in these patients $(53,54)$. A study found that the CLASI score correlated with the IFN score, therefore correlating with disease activity (54). Another study using BIIB059, an anti-BDCA2 monoclonal antibody, found correlations between CLASI scores and cutaneous LE biomarkers (55). Administration of BIIB059 in SLE patients decreased $M x 1$, a type I interferon-upregulated protein, as well as inflammatory cells found in active disease, which was also reflected in the reduction of the CLASI activity score (55). The CLASI is a valid instrument when conducting clinical trials, especially given its ability to correlate scores with physiologic improvements in patients following drug administration.

\section{CLE and QoL}

CLE is a chronic condition that currently can be managed but not cured (3). Because CLE tends to present as lesions on visible surface areas such as the face and arms, CLE is often associated with vocational disability (56). Compared to other dermatologic conditions, like acne, non-melanoma skin cancer, and alopecia, CLE patients have worse QoL (3). Compared to patients with hypertension, type 2 diabetes mellitus, recent myocardial infarction, and congestive heart failure, CLE patients have worse QoL, particularly within the parameters of emotional health (3). The negative impact that CLE has on patients' lives demonstrates the need for therapies that improve the disease; improvement in QoL should be one of the main objectives when treating these patients.

In patients taking antimalarial and antimetabolite medications, an improvement in disease activity resulted in an improvement in QoL, even if the disease did not entirely subside (8). Medications that meaningfully improve QoL may not exhibit near or complete clearance of disease activity but still be an important improvement for disease management (57). Several studies using the CLASI have assessed potential therapeutic options and found promising results $(12-14,42-44,55,58,59)$.

Regulatory guidance on endpoints for measuring skin activity in SLE and CLE patients is essential to improve therapies. Individual and aggregate CLASI activity scores 
measure specific areas of activity, and correlate with QoL before and after treatment (3). In a study validating the CLASI in a pediatric population, it was confirmed that children who had a reduction in the CLASI activity score by at least $50 \%$ had a significantly improved QoL, as measured by the Pediatric Quality of Life Inventory Generic Core scale (PedsQL-GC) and the pediatric adaptation of the Skindex-29 (pSkindex27) (39). These findings support that a $50 \%$ improvement in CLASI (CLASI50) activity is clinically meaningful and can be used to determine a significant response to therapy despite not achieving complete clearance of their disease activity (60).

In addition, Klein et al. identified a four-point or $20 \%$ decrease in CLASI activity as minimal clinically significant improvement in the CLASI activity score (30). However, for meaningful reduction based on assessment of QoL, a 6-point decrease or $50 \%$ reduction in CLASI activity for patients with an initial activity score of 8 or higher reflect an important change (60). Either a raw change or percent improvement can be used effectively to determine meaningful change. While complete remission is a score of zero, it is known that for CLASI activity scores $\leq 3$, there is less impact on a patient's QoL (57).

\section{The use of CLASI in clinical trials and emerging therapies for lupus}

Several therapeutic interventions are currently used to treat CLE. Although the majority of these interventions are typically well-tolerated and can be effective, those used for refractory disease can cause serious side effects (8). Despite this, in the past fifty years, only one new drug has been approved for SLE and no drug has been approved for CLE $(8,61)$. This lack of advancement is largely associated with the design and results of the clinical trials $(8,33)$. For instance, many randomized control trials comparing a potential treatment to placebo have shown high placebo response rates, at times due to the effects of background therapy such as oral steroids (8). Furthermore, lupus drug trials have often excluded patients with CLE and traditionally focused on those with SLE, making it a challenge to truly assess the efficacy of therapeutic interventions for CLE $(8,61)$. The development of the CLASI has made it possible to evaluate the treatment of cutaneous disease in SLE as well as CLE, providing a more consistent outcome measure and quantifiable endpoint $(8,61)$.

The CLASI has been used in multiple large clinical trials, and the results have been promising for the further development of CLE treatment. For patients with at least moderate skin involvement (CLASI $\geq 10$ ), clinical trials endpoints currently use a percentage of patients with $\geq 50 \%$ improvement in CLASI activity or a percentage of patients with at least a 4-point decrease in CLASI activity, which represents a clinically significant improvement (12-14,30).

A recent study investigated the effect of AMG 811, a monoclonal antibody that blocks interferon-gamma (IFN) (62). Although treatment with AMG 811 led to changes in the IFN $\gamma$ levels found in the blood and skin of patients with DLE, there was no clinical benefit detected by the CLASI, the physician's assessment of skin disease or the patient's self-assessment of their skin disease (62). Thus, the CLASI is correlated with self-reported patient outcomes. It is not only effective in reflecting an improvement in disease activity, but it is also effective when demonstrating that a therapeutic intervention does not provide clinical benefit.

A trial studying the effects of sifalimumab, an antiIFN $\alpha$ antibody, using an efficacy measure of a 4-point reduction in CLASI for patients with at least moderate severity (CLASI $\geq 10$ ), found that a greater percentage of patients treated with sifalimumab had a larger improvement in CLASI compared to the placebo (13). Similarly, using an efficacy measure of percentage of patients with $\geq 50 \%$ improvement in CLASI for patients with at least moderate skin involvement (CLASI $\geq 10)$, treatment in a phase 2 and phase 3 trial with anifrolumab, an anti-IFN- $\alpha$ receptor monoclonal antibody, resulted in a higher percentage of patients with skin improvement compared to the placebo $(12,63)$. In both studies, the CLASI score correlated with improvement in cutaneous disease, further demonstrating the importance of using this outcome measure for future clinical trials.

Additional studies examining the clinical effects of emerging therapies have also been performed, many of which have shown promising results. Treatment with BIIB059, an anti-BDCA2 monoclonal antibody, was found to decrease the CLASI activity score compared to the placebo in SLE patients with active manifestations of ACLE, SCLE and/or CCLE (55). CC-220, a CUL4CRBN $\mathrm{E} 3$ ubiquitin ligase modulator that reduces the transcription factors Ikaros (IKZF1) and Aiolos (IKZF3), resulted in an improvement in CLASI activity score in all treatment groups compared to the placebo, with reductions in CLASI activity score more significant in patients with moderate-tosevere skin involvement (59). The improvement in CLASI 
activity score in patients treated with CC-220 also showed a strong correlation in plasmacytoid dendritic cell (pDC) depletion, further emphasizing the validity of the CLASI score as a reliable tool to determine clinical and physiologic disease improvement (59).

\section{Summary}

The CLASI is the only clinical outcome measure that is specific to CLE that demonstrates content and construct validity, inter-rater validity, intra-rater validity and practical applicability $(27,37,38,40,41)$. It allows for the documentation of treatment impact on disease activity and monitors change in disease progression. Its use in clinical trials has demonstrated that the CLASI is responsive to change in disease, both clinically and physiologically following therapeutic intervention.

The CLASI can be applied to a multitude of CLE subtypes, except for the rare manifestations of lupus panniculitis and bullous lupus. Patients often have a diagnosis of more than one subtype, and it is important to monitor disease activity and damage of the various morphologies with one disease index to have a greater understanding of overall disease progression.

Extensive studies of the CLASI have resulted in several known features that are used in clinical trials. The CLASI can be used to categorize patients based on severity, which can provide cutoff values for study inclusion (30). In addition, a 4-point change in disease activity can be used to identify patients with clinically significant improvement (30). The CLASI is not only used to capture disease progression but can also be correlated to changes in QoL (3). The responsiveness of the CLASI has also been validated with interventional studies (42-51).

There have been a number of early trials for CLE and SLE that have utilized the CLASI as a primary or secondary endpoint and demonstrated meaningful differences between treatment and placebo. Many current trials exclude patients with CLE that don't meet criteria for SLE, denying these patients access to potential new therapies. Using the CLASI in these trials would allow for the evaluation of disease activity on the skin and provide an interpretable and meaningful endpoint for organ-specific trials of cutaneous lupus that include patients with CLE and/or SLE.

In the past 50 years, there have been no new FDA approved treatments for CLE, a disease known to significantly impair a patient's QoL. While novel treatments may not result in the complete clearance of cutaneous disease activity, they have been proven to demonstrate a meaningful impact on patients' QoL. The CLASI is vital for the progression of clinical trials, which are necessary for the advancement of new treatments for lupus.

\section{Acknowledgments}

Funding: United States Department of Veterans Affairs (Veterans Health Administration, Office of Research and Development and Biomedical Laboratory Research and Development) (VPW) and National Institutes of Health (National Institute of Arthritis and Musculoskeletal and Skin Diseases) R01AR071653 (VPW).

\section{Footnote}

Provenance and Peer Review: This article was commissioned by the editorial office, Annals of Translational Medicine, for the series "Rheumatologic Skin Disease". The article has undergone external peer review.

Reporting Checklist: The authors have completed the Narrative Review reporting checklist. Available at http:// dx.doi.org/10.21037/atm-20-5048

Conflicts of Interest: All authors have completed the ICMJE uniform disclosure form (available at http://dx.doi. org/10.21037/atm-20-5048). The series "Rheumatologic Skin Disease" was commissioned by the editorial office without any funding or sponsorship. VPW served as the unpaid Guest Editor of the series. Dr. BFC reports grants from Daavlin Corporation, other from Pfizer Corporation, other from Biogen Corporation, personal fees from Bristol Meyers Squibb, personal fees from Viela Bio, personal fees from Beacon Bioscience, during the conduct of the study. Dr. JFM reports personal fees from Abbvie, personal fees from Dermavant, personal fees from Eli Lilly, personal fees from Novartis, personal fees from Janssen, personal fees from UCB, personal fees from Celgene, personal fees from Sanofi Regeneron, personal fees from Arena, personal fees from Sun Pharma, personal fees from Biogen, personal fees from Pfizer, personal fees from EMD Sorono, personal fees from Avotres, personal fees from Leo Pharma, personal fees from CARA Therapeutics, outside the submitted work. Dr. VPW reports personal fees from Genentech, grants and personal fees from Biogen, personal fees from Medimmune, grants and personal fees from Gilead, personal fees from Amgen, personal fees from Resolve, grants and personal 
fees from Celgene, personal fees from Lilly, personal fees from Principia, personal fees from BMS, personal fees from ACI, grants and personal fees from Janssen, personal fees from Nektar, personal fees from EMD Serona, personal fees from Astra Zeneca, personal fees from Vielo, personal fees from Abbvie, personal fees from Kyowa Kirin, personal fees from GSK, personal fees from Cugene, outside the submitted work; in addition, Dr. VPW has a patent CLASI with royalties paid, and a patent CDASI with royalties paid. The authors have no other conflicts of interest to declare.

Ethical Statement: The authors are accountable for all aspects of the work in ensuring that questions related to the accuracy or integrity of any part of the work are appropriately investigated and resolved.

Open Access Statement: This is an Open Access article distributed in accordance with the Creative Commons Attribution-NonCommercial-NoDerivs 4.0 International License (CC BY-NC-ND 4.0), which permits the noncommercial replication and distribution of the article with the strict proviso that no changes or edits are made and the original work is properly cited (including links to both the formal publication through the relevant DOI and the license). See: https://creativecommons.org/licenses/by-nc-nd/4.0/.

\section{References}

1. Patel P, Werth V. Cutaneous lupus erythematosus: a review. Dermatol Clin 2002;20:373-85, v.

2. Werth VP. Clinical manifestations of cutaneous lupus erythematosus. Autoimmun Rev 2005;4:296-302.

3. Klein R, Moghadam-Kia S, Taylor L, et al. Quality of life in cutaneous lupus erythematosus. J Am Acad Dermatol 2011;64:849-58.

4. Moghadam-Kia S, Chilek K, Gaines E, et al. Crosssectional analysis of a collaborative Web-based database for lupus erythematosus-associated skin lesions: prospective enrollment of 114 patients. AMA Arch Derm 2009;145:255-60.

5. Chang AY, Werth VP. Treatment of cutaneous lupus. Current rheumatology reports 2011;13:300-7.

6. Okon LG, Werth VP. Cutaneous lupus erythematosus: diagnosis and treatment. Best Pract Res Clin Rheumatol 2013;27:391-404.

7. Mittal L, Zhang L, Feng R, et al. Antimalarial drug toxicities in patients with cutaneous lupus and dermatomyositis: A retrospective cohort study. J Am Acad
Dermatol 2018;78:100-6.e1.

8. Presto JK, Werth VP. Cutaneous Lupus Erythematosus: Current Treatment Options. Curr Treatm Opt Rheumatol 2016;2:36-48.

9. Caplan A, Fett N, Rosenbach M, et al. Prevention and management of glucocorticoid-induced side effects: a comprehensive review: gastrointestinal and endocrinologic side effects. J Am Acad Dermatol 2017;76:11-6.

10. Caplan A, Fett N, Rosenbach M, et al. Prevention and management of glucocorticoid-induced side effects: a comprehensive review: a review of glucocorticoid pharmacology and bone health. J Am Acad Dermatol 2017;76:1-9.

11. US Department of Health and Human Services FDA CfDEaRC. Guidance for industry: systemic lupus erythematosus: developing drugs for treatment. 2010.

12. Furie R, Khamashta M, Merrill JT, et al. Anifrolumab, an Anti-Interferon-alpha Receptor Monoclonal Antibody, in Moderate-to-Severe Systemic Lupus Erythematosus. Arthritis Rheumatol 2017;69:376-86.

13. Khamashta M, Merrill JT, Werth VP, et al. Safety and Efficacy of Sifalimumab, an Anti Ifn-alpha Monoclonal Antibody, in a Phase 2b Study of Moderate to Severe Systemic Lupus Erythematosus (SLE). 14. Arthritis Rheumatol 2014;66:3530-1.

14. van Vollenhoven RF, Hahn BH, Tsokos GC, et al. Efficacy and safety of ustekinumab, an IL-12 and IL-23 inhibitor, in patients with active systemic lupus erythematosus: results of a multicentre, double-blind, phase 2, randomised, controlled study. Lancet 2018;392:1330-9.

15. Bombardier C, Gladman DD, Urowitz MB, et al. Derivation of the SLEDAI. A disease activity index for lupus patients. The Committee on Prognosis Studies in SLE. Arthr Rheum 1992;35:630.

16. Urowitz MB, Gladman DD, Tozman EC, et al. The lupus activity criteria count (LACC). J Rheumatol 1984;11:783.

17. Gladmann DD, Urowitz MB, Ong A, et al. A comparison of five health status instruments in patients with systemic lupus erythematosus (SLE). Lupus 1996;5:190.

18. Parodi A, Massone C, Cacciapuoti M, et al. Measuring the activity of the disease in patients with cutaneous lupus erythematosus. Br J Dermatol 2000;142:457.

19. Ahmad Fadzil MH, Ihtatho D, Mohd Affandi A, et al. Objective assessment of psoriasis erythema for PASI scoring. J Med Eng Technol 2009;33:516-24.

20. Leshem YA, Hajar T, Hanifin JM, et al. What the Eczema Area and Severity Index score tells us about the severity of atopic dermatitis: an interpretability study. Br J Dermatol 
2015;172:1353-7.

21. Faust HB, Gonin R, Chuang TY, et al. Reliability testing of the dermatology index of diseease severity (DIDS). An index for staging the severity of cutaneous inflammatory disease. Arch Dermatol 1997;133:1443.

22. Albrecht J, Werth VP. Outcome Measures in Cutaneous Autoimmune Disease: Dermatomyositis and Lupus Erythematosus. The Skin in Systemic Autoimmune Disease. Elsevier; 2006.

23. Albrecht J, Werth VP. Development of the CLASI as an outcome instrument for cutaneous lupus erythematosus. Dermatol Ther 2007;20:93-101.

24. Walling HW, Sontheimer RD. Cutaneous lupus erythematosus. Am J Clin Dermatol 2009;10:365-81.

25. Grönhagen CM, Nyberg F. Cutaneous lupus erythematosus: An update. Indian Dermatol Online J 2014;5:7-13.

26. Watanabe T, Tsuchida T. Classification of lupus erythematosus based upon cutaneous manifestations. Dermatological, systemic and laboratory findings in 191 patients. Dermatology 1995;190:277-83.

27. Albrecht J, Taylor L, Berlin JA, et al. The CLASI (Cutaneous Lupus Erythematosus Disease Area and Severity Index): an outcome instrument for cutaneous lupus erythematosus. J Invest Dermatol 2005;125:889-94.

28. Verma SM, Okawa J, Propert KJ, et al. The impact of skin damage due to cutaneous lupus on quality of life. Br J Dermatol 2014;170:315-21.

29. Vasquez R, Wang D, Tran Q, et al. A multicentre, crosssectional study on quality of life in patients with cutaneous lupus erythematosus. Br J Dermatol 2013;168:145-53.

30. Klein R, Moghadam-Kia S, LoMonico J, et al. Development of the CLASI as a tool to measure disease severity and responsiveness to therapy in cutaneous lupus erythematosus. Arch Dermatol 2011;147:203-8.

31. Schäfer I, Hacker J, Rustenbach SJ, et al. Concordance of the Psoriasis Area and Severity Index (PASI) and patientreported outcomes in psoriasis treatment. Eur J Dermatol 2010;20:62-7.

32. Fadzil MA, Ihtatho D. editors. Modeling psoriasis lesion colour for PASI erythema scoring. In: Information Technology, 2008. ITSim 2008. International Symposium on 2008;2:1-6.

33. Merrill JT, Manzi S, Aranow C, et al. Lupus community panel proposals for optimising clinical trials: 2018. Lupus Sci Med 2018;5:e000258.

34. Lahti A, Kopola H, Harila A, et al. Assessment of skin erythema by eye, laser Doppler flowmeter, spectroradiometer, two-channel erythema meter and Minolta chroma meter. Arch Dermatol Res 1993;285:278-82.

35. Zhao C, Hao E, Oh D, et al. A comparison study of clinician-rated atopic dermatitis outcome measures for intermediate-to dark-skinned patients. Br J Dermatol 2017;176:985-92.

36. AlE'ed A, Aydin POA, Al Mutairi N, et al. Validation of the Cutaneous Lupus Erythematosus Disease Area and Severity Index and pSkindex27 for use in childhoodonset systemic lupus erythematosus. Lupus Sci Med 2018;5:e000275.

37. Krathen M, Dunham J, Gaines E, et al. The cutaneous lupus erythematosus disease activity and severity index: expansion for rheumatology and dermatology. Arthritis Rheum 2008;59:338-44.

38. Kushner CJ, Tarazi M, Gaffney RG, et al. Evaluation of the reliability and validity of the Cutaneous Lupus Erythematosus Disease Area and Severity Index (CLASI) in paediatric cutaneous lupus among paediatric dermatologists and rheumatologists. Br J Dermatol 2019;180:165-71.

39. Aydin POA, Al Mutairi N, AlSaleem A, et al. Validation of the Cutaneous Lupus Erythematosus Disease Area and Severity Index and pSkindex27 for use in childhoodonset systemic lupus erythematosus. Lupus Sci Med 2018;5:e000275.

40. Jolly M, Kazmi N, Mikolaitis RA, et al. Validation of the Cutaneous Lupus Disease Area and Severity Index (CLASI) using physician- and patient-assessed health outcome measures. J Am Acad Dermatol 2013;68:618-23.

41. Bonilla-Martinez ZL, Albrecht J, Troxel AB, et al. The cutaneous lupus erythematosus disease area and severity index: a responsive instrument to measure activity and damage in patients with cutaneous lupus erythematosus. AMA Arch Derm 2008;144:173-80.

42. Kreuter A, Gaifullina R, Tigges C, et al. Lupus erythematosus tumidus: response to antimalarial treatment in 36 patients with emphasis on smoking. AMA Arch Derm 2009; 145:244-8.

43. Kreuter A, Tomi N, Weiner S, et al. Mycophenolate sodium for subacute cutaneous lupus erythematosus resistant to standard therapy. Br J Dermatol 2007;156:1321-7.

44. Erceg A, Bovenschen HJ, van de Kerkhof PC, et al. Efficacy and safety of pulsed dye laser treatment for cutaneous discoid lupus erythematosus. J Am Acad Dermatol 2009;60:626-32. 
45. Chang AY, Piette EW, Foering KP, et al. Response to antimalarial agents in cutaneous lupus erythematosus: a prospective analysis. AMA Arch Derm 2011;147:1261-7.

46. Ikeda T, Kanazawa N, Furukawa F. Hydroxychloroquine administration for Japanese lupus erythematosus in Wakayama: a pilot study. J Dermatol 2012;39:531-5.

47. Yokogawa N, Tanikawa A, Amagai M, et al. Response to hydroxychloroquine in Japanese patients with lupus-related skin disease using the cutaneous lupus erythematosus disease area and severity index (CLASI). Mod Rheumatol 2013;23:318-22.

48. Cortés-Hernández J, Ávila G, Vilardell-Tarrés M, et al. Efficacy and safety of lenalidomide for refractory cutaneous lupus erythematosus. Arthritis Res Ther 2012;14:R265.

49. Cortés-Hernández J, Torres-Salido M, Castro-Marrero J, et al. Thalidomide in the treatment of refractory cutaneous lupus erythematosus: prognostic factors of clinical outcome. Br J Dermatol 2012;166:616-23.

50. De AS, Strober BE, Merola JF, et al. Apremilast for discoid lupus erythematosus: results of a phase 2, open-label, single-arm, pilot study. J Drugs Dermatol 2012;11:1224-6.

51. Ky C, Swasdibutra B, Khademi S, et al. Efficacy of intravenous immunoglobulin monotherapy in patients with cutaneous lupus erythematosus: results of proof-ofconcept study. Dermatol Reports 2015;7:5804.

52. Concha JSS, Patsatsi A, Marshak-Rothstein A, et al. Advances in Cutaneous Lupus Erythematosus and Dermatomyositis: A Report from the 4th International Conference on Cutaneous Lupus ErythematosusAn Ongoing Need for International Consensus and Collaborations. J Invest Dermatol 2019;139:270-6.

53. Oh SH, Roh HJ, Kwon JE, et al. Expression of interleukin-17 is correlated with interferon- $\alpha$ expression in cutaneous lesions of lupus erythematosus. Clin Exp Dermatol 2011;36:512-20.

54. Braunstein I, Klein R, Okawa J, et al. The interferonregulated gene signature is elevated in subacute cutaneous lupus erythematosus and discoid lupus erythematosus and correlates with the cutaneous lupus area and severity index score. Br J Dermatol 2012;166:971-5.

Cite this article as: Chakka S, Krain RL, Concha JSS, Chong BF, Merola JF, Werth VP. The CLASI, a validated tool for the evaluation of skin disease in lupus erythematosus: a narrative review. Ann Transl Med 2021;9(5):431. doi: 10.21037/ atm-20-5048
55. Furie R, Werth VP, Merola JF, et al. BIIB059, an antiBDCA2 mAb, ameliorates skin lesions in SLE. J Clin Invest 2019;129:1359-71.

56. Smolen JS, Weinblatt ME, Sheng S, et al. Sirukumab, a human anti-interleukin-6 monoclonal antibody: a randomised, 2-part (proof-of-concept and dose-finding), phase II study in patients with active rheumatoid arthritis despite methotrexate therapy. Ann Rheum Dis 2014;73:1616-25.

57. Tarazi M, Gaffney R, Feng R, et al. Evaluating cutaneous lupus disease activity end points and their effects on quality of life as an outcome measure for clinical trials. $\mathrm{Br}$ J Dermatol 2019;181:841-2.

58. Furie R, Werth V, Merola J, et al. 83 Biib059, a monoclonal antibody targeting bdca2, demonstrates evidence of proof of biological activity in subjects with cutaneous lupus. Archives of Disease in Childhood; 2017.

59. Werth VP, Furie R, Gaudy A, et al. editors. CC-220 Decreases B-Cell Subsets and Plasmacytoid Dendritic Cells in Systemic Lupus Erythematosus (SLE) Patients and Is Associated with Skin Improvement: Pharmacodynamic Results from a Phase IIa Proof of Concept Study. Arthritis Rheumatol; 2017: Wiley 111 River ST, Hoboken 070305774, NJ, USA.

60. Chakka S, Krain RL, Ahmed S, et al. Evaluating change in disease activity needed to reflect meaningful improvement in quality of life for clinical trials in cutaneous lupus erythematosus. J Am Acad Dermatol 2020. [Epub ahead of print].

61. Presto JK, Hejazi EZ, Werth VP. Biological therapies in the treatment of cutaneous lupus erythematosus. Lupus 2017;26:115-8.

62. Werth VP, Fiorentino D, Sullivan BA, et al. Brief Report: Pharmacodynamics, Safety, and Clinical Efficacy of AMG 811, a Human Anti-Interferon- $\gamma$ Antibody, in Patients With Discoid Lupus Erythematosus. Arthritis Rheumatol 2017;69:1028-34.

63. Morand EF, Furie R, Tanaka Y, et al. Trial of anifrolumab in active systemic lupus erythematosus. $\mathrm{N}$ Engl J Med 2020;382:211-21. 